\title{
Long-Term Survival of a Patient with Glioblastoma
}

\author{
Adrian Sacher, Robert Wollmann, Urmi Kalokhe, David Munoz, Marie-Christine Guiot, \\ R. Loch Macdonald
}

Can. J. Neurol. Sci. 2010; 37: 289-292

Glioblastoma (GB) accounts for $50-60 \%$ of all intracranial gliomas and has a median survival of 13.6 months even with aggressive surgical resection, radiation and chemotherapy ${ }^{1}$. This dismal prognosis is compounded by the failure of novel therapies to yield more than minimal prognostic improvements. A major advance was adjuvant temozolamide treatment but this increased median survival in multi-centre trials by only 2.5 months $^{2-4}$.

Prognostic indicators have been identified for GB and include young age, female sex, good Karnofsky performance status, absence of focal neurological deficits and complete surgical resection ${ }^{1,5-9}$. Histological characteristics such as the presence of oligodendroglial components and giant cells have also been associated with improved survival ${ }^{7,9,10}$. Genetic and chromosomal alterations have been examined as prognostic indicators and therapeutic targets. Methyl-guanine-methyl-transferase (MGMT) hypermethylation is the best established of these and predicts both survival and response to alkylating agents including procarbazine/lomustine/vincristine and temozolamide $^{2,7}$. Less well accepted markers of favourable prognosis include p53 mutation, epidermal growth factor receptor amplification and losses of chromosomes $1 p$ and $19 q^{8,11-13}$ whereas controversial markers of poor prognosis include gains of chromosome 7 , loss of chromosome $9 \mathrm{p} / 10 \mathrm{p} / 10 \mathrm{q}$ and $\mathrm{mdm} 2$ overexpression $^{8,11,12}$.

Despite a poor prognosis, $2 \%$ of patients with GB survive more than three years ${ }^{5,6,14-16}$. It might be expected that these patients would have identifiable molecular markers; however, no distinct markers consistently present in long-term survivors have been established and the mechanism behind the improved survival remains unknown.

\section{Case Report}

A 49-year-old male presented with a three-month history or worsening ataxia and nausea. On neurological examination, the patient had mild right upper limb intention tremor and dysdiadochokinesis. Magnetic resonance imaging (MRI) revealed an irregular, contrast-enhancing mass in the right cerebellar hemisphere measuring about $4 \mathrm{~cm}$ in diameter (Figure 1). A paramedian suboccipital craniectomy was performed and resection attempted. The mass was markedly vascular and abutted on the tentorium superiorly. The other margins were quite well defined but after partial resection and loss of several liters of blood, the resection was aborted. The patient was sedated and intubated overnight and resection was completed relatively easily the next day. Complete resection was confirmed by absence of enhancement around the postoperative resection cavity on MRI one day later.
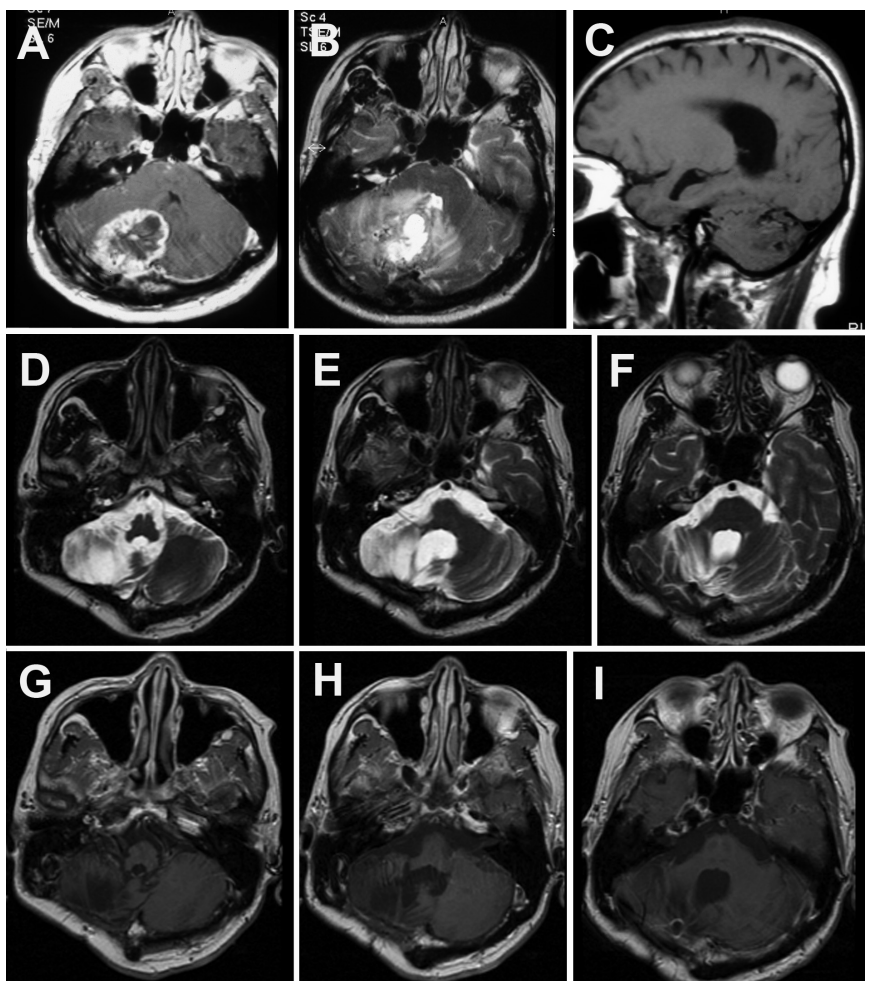

Figure 1: Preoperative axial T1 weighted with gadolinium (A), T2 axial $(B)$ and $T 1$ sagittal $(C)$ without gadolinium magnetic resonance images (MRI) show an irregular, enhancing intraaxial right cerebellar mass with surrounding hyperintensity on $T 2$ image and prominent flow voids on the sagittal image. A series of axial T2 (D-F) and T1 (G-I) MRIs with gadolinium obtained eight years after surgical resection, radiation and chemotherapy show an area of encephalomalacia but no enhancement showing no evidence of tumor recurrence.

From the Divisions of Neurosurgery (AS, RLM), Pathology (DM), Keenan Research Centre, Li Ka Shing Knowledge Institute, St. Michael's Hospital, Department of Surgery, University of Toronto, Toronto, Ontario, Canada; Department of Pathology, University of Chicago Medical Center and the Pritzker School of Medicine (RW), Chicago, Illinois, USA; St. Margaret Mercy Hospital (UK), Hammond, Indiana, USA; Department of Pathology (MCG), McGill University, Montreal, Quebec, Canada.

Received February 23, 2009. Final Revisions Submitted September 24, 2009. Correspondence to: R. Loch Macdonald, Division of Neurosurgery, St. Michael's Hospital, 30 Bond Street, Toronto, Ontario, M5B 1W8, Canada. 


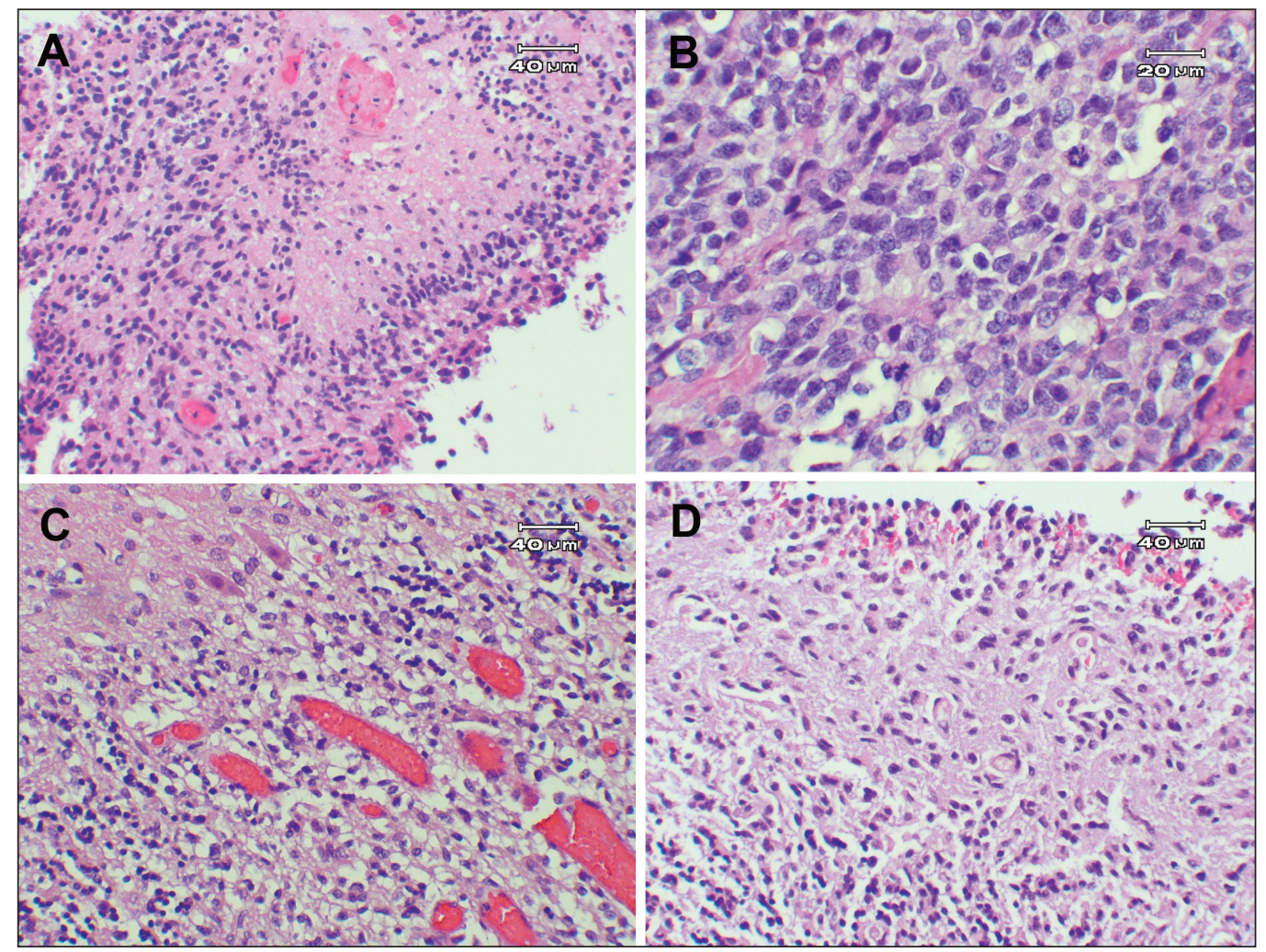

Figure 2: Histopathology of GB with long-term survival shows necrosis with pseudopallisading (A) and prominent mitotic activity $(B)$. Anaplastic astrocytes also can be seen infiltrating into the internal granule cell $(C)$ and molecular layers $(D)$ of the cerebellum (hematoxylin and eosin).

Histology revealed a highly cellular infiltrative glial neoplasm with moderate nuclear pleomorphism, several multinucleated forms, numerous mitoses and multiple foci of necrosis and endothelial hyperplasia. There were occasional perinuclear halos and variable amounts of eosinophilic cytoplasm (Figure 2). There was immunoreactivity to glial fibrillary acidic protein but not to S100. The Ki67 labeling index was estimated to be $20 \%$. A pathological diagnosis of GB with subtle oligodendroglial features was made, although the tumor was thought to be a GB and not an anaplastic oligodendroglioma. The patient had fractionated external beam radiotherapy performed based on computed tomographic simulation fused with the preoperative MRI. The target volume ( 10.5 by 12 by $7 \mathrm{~cm}$ ) was defined to include the tumor volume on the preoperative MRI plus a 1.5 to $2 \mathrm{~cm}$ margin. This volume was treated with $4800 \mathrm{cGy}$ in 24200 cGy fractions administered five days per week. After this a boost volume ( 8.5 by 9.5 by $6.5 \mathrm{~cm}$ ) with a reduced margin was treated to a total dose of $6000 \mathrm{cGy}$ in 30 fractions over six weeks. A three dimensional conformal plan with three-field arrangement of right posterior, left posterior and right anterior oblique portals of 6 and $18 \mathrm{MV}$ photons was used for the entire treatment. This was followed by an 18-month course of chemotherapy with procarbazine, lomustine and vincristine. Recovery was complete and the patient returned to an active practice of surgery.
Follow-up over the next eight years showed no enhancement at the resection site and no change in hyperintensity on T2 and FLAIR images. The patient did not manifest any clinical or radiologic evidence of recurrence (Figure 1). Seven and a half years after surgery he developed biopsy proven prostate carcinoma which was local and has not been treated yet.

Five years after the surgical recurrence, the long recurrencefree survival prompted review of the pathology by the same pathologist who did not know the patient's condition but the diagnosis remained GB. Independent pathologic review confirmed this. Fluorescence in-situ hybridization analysis of the patient's tumor also was performed on stored paraffin-embedded tissue. Human RPC1-11 BAC library-derived probes (Research Genetics, Huntsville, AL) specific for chromosomal regions $1 \mathrm{p} 32,1 \mathrm{q} 42,19 \mathrm{p} 13$, and $19 \mathrm{q} 13$ were used to detect loss of heterozygosity at chromosomes $1 \mathrm{p}$ and $19 \mathrm{q}$. The tumor was found to possess normal $1 \mathrm{p}$ dosage and loss of heterozygosity at chromosome 19q. Immunohistochemistry for O6 methylguanine-DNA methyltransferase (MGMT) did not demonstrate positive staining.

O-6-methylguanine-DNA methyltransferase was also assessed by polymerase chain reaction $(\mathrm{PCR})^{17}$. Genomic DNA was isolated from $10 \mu \mathrm{m}$ thick paraffin sections using a QIAamp DNA mini kit (Qiagen Sciences, Maryland, USA). Bisulfite treatment of $1 \mu \mathrm{g}$ of DNA was performed using EZ DNA 


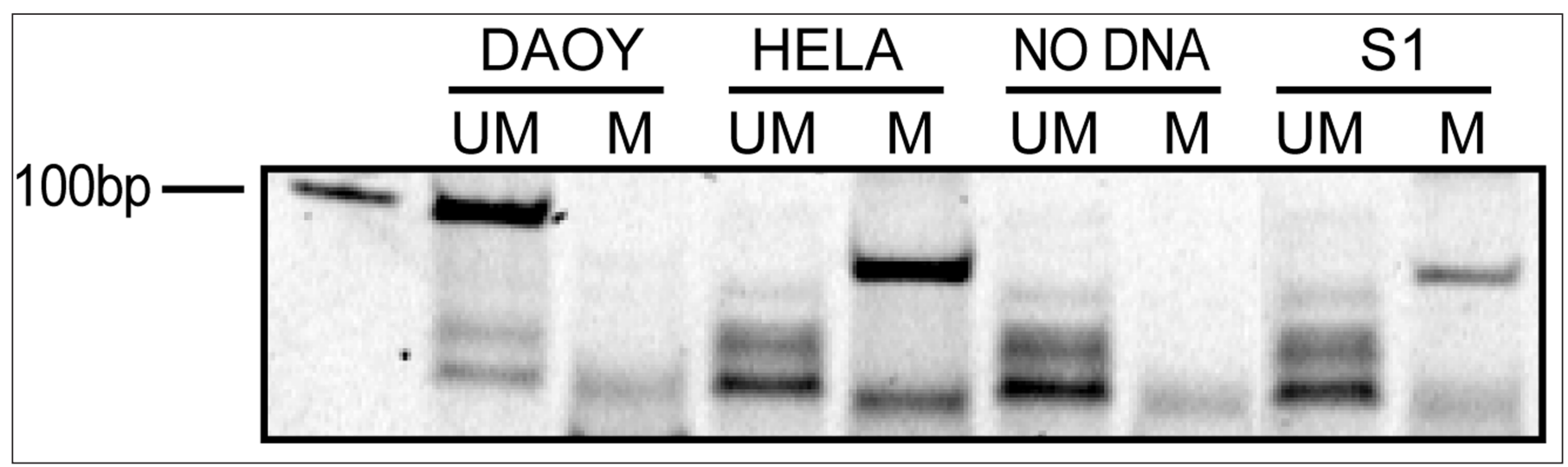

Figure 3: MGMT methylation by PCR. DAOY cells were unmethylated (UM) whereas HELA cells and the tumor sample (S1) show methylation (M).

Methylation Gold Kit (Zymo Research, Orange, CA, USA). Methylation specific polymerase chain reaction was performed using specific primers to distinguish methylated and unmethylated DNA. Sequences of primers were: unmethylated forward 5'TTTGTGTTTTGATGTTTGTAGGTTTTTGT 3', unmethylated reverse 5'AACTCCACACTCTTCCAAAAACA AAACA 3'; methylated forward 5' TTTCGACGTTCGTAGG TTTTCGC 3'; methylated reverse GCACTCTTCCGAAAAC GAAACG 3'. Methylated and unmethylated PCR products were visualized on a Criterion precast gel 10\% TBE (Bio-Rad, Hercules, CA, USA). Control DNA was treated concomitantly and consisted of genomic DNA isolated from Hela cells for the methylated form and from DAOY cells for the unmethylated form. This showed that MGMT was methylated (Figure 3).

\section{Discussion}

Long-term survivors of GB constitute rare exceptions to the dismal prognosis otherwise associated with this tumor. The study of these patients may provide an understanding of how to both predict and prolong survival of patients with GB. Factors contributing to long-term survival include patient characteristics, histological attributes, treatments and genetics. However, the infrequent occurrence of such survivors and the heterogeneity of their tumors have not led to definition of any specific characteristic associated with long-term survival.

The patient described here is a rare example of eight year progression-free survival following GB resection and adjuvant therapy. Superficially, this patient possessed many characteristics associated with good prognosis including young age, good pre-morbid functioning and gross total resection. Some histological features present in this case also have been suggested to improve prognosis including the presence of giant cells and oligodendroglial features. However, the other features were the loss of heterozygosity of chromosome $19 q$ without loss at $1 \mathrm{p}$ and methylation of MGMT.

Combined loss of heterozygosity of chromosome $1 p$ and $19 q$ was originally found to be a useful marker of survival and susceptibility to alkylating agents in oligodendroglioma, oligoastrocytoma and oligodendroglial components of $\mathrm{GB}^{4,18-22}$.
The prognostic significance of combined loss of $1 p$ and $19 q$ in patients with GB, however, is controversial with studies both refuting and supporting its role in prognosis ${ }^{8,11,13,23}$. It also is controversial whether tumors that resemble GB histologically but have combined loss of $1 \mathrm{p}$ and $19 \mathrm{q}$ should be considered GB or anaplastic oligodendroglioma ${ }^{24}$. Losses of specific regions chromosome 1p appear to predict improved survival in GB particularly in the centromeric regions associated with the NOTCH2 gene 23,25 . The impact of a solitary loss of heterozygosity at $19 q$ remains unclear as few studies have considered its impact on survival independent of $1 \mathrm{p}$ loss. However, it is clear that loss of heterozygosity at $19 q$ occurs much more commonly among long-term survivors of GB and that it often occurs without $1 \mathrm{p}$ loss ${ }^{11,23}$.

There are some limitations to the single locus technology used here to assess loss of heterozygosity. It is possible that partial allelic loss of $1 \mathrm{p}$ could have been missed. Polymerase chain reaction-based techniques using a larger number of probes, or technology such as CGH might demonstrate loss of $1 \mathrm{p}$, which would suggest the tumor arose from oligodendroglia. The routine microscopy of the tumor also suggested some features of oligodendroglial lineage. Glioblastomas with oligodendroglial features, loss of $1 \mathrm{p}$ and $19 \mathrm{q}$ are described and associated with longer survival ${ }^{24}$.

This tumor also did not have immunoreactivity to MGMT but the more specific PCR reaction did show methylation of MGMT. Epigenetic silencing of MGMT by methylation of the promoter is associated with response to temozolamide chemotherapy in patients with malignant astrocytoma and glioblastoma ${ }^{2}$. It also may be associated with longer survival in patients who are treated with other types of chemotherapy ${ }^{26}$.

Another unique feature of this case is location of the GB in the cerebellum. Only $1 \%$ of GB are located in the cerebellum $^{27,28}$. Why they are less common here than would be expected based on brain volume, and to some extent astrocyte numbers, is unknown. A brief review of the literature suggests that cerebellar GB have similar male predominance, peak age incidence in sixth decade of life, propensity for local recurrence and similar poor prognosis to supratentorial $\mathrm{GB}^{27-29}$. 


\section{REFERENCES}

1. Filippini G, Falcone C, Boiardi A, Broggi G, Bruzzone MG, Caldiroli D, et al. Prognostic factors for survival in 676 consecutive patients with newly diagnosed primary glioblastoma. Neuro Oncol. 2008;10:79-87.

2. Hegi ME, Diserens AC, Gorlia T, Hamou MF, de TN, Weller M, et al. MGMT gene silencing and benefit from temozolomide in glioblastoma. N Engl J Med. 2005;352:997-1003.

3. Stupp R, Mason WP, van den Bent MJ, Weller M, Fisher B, Taphoorn MJ, et al. Radiotherapy plus concomitant and adjuvant temozolomide for glioblastoma. N Engl J Med. 2005;352: 987-96.

4. van den Bent MJ, Hegi ME, Stupp R. Recent developments in the use of chemotherapy in brain tumours. Eur J Cancer. 2006;42: $582-8$

5. Scott JN, Rewcastle NB, Brasher PM, Fulton D, Hagen NA, MacKinnon JA, et al. Long-term glioblastoma multiforme survivors: a population-based study. Can J Neurol Sci. 1998;25: 197-201.

6. Scott JN, Rewcastle NB, Brasher PM, Fulton D, MacKinnon JA, Hamilton M, et al. Which glioblastoma multiforme patient will become a long-term survivor? A population-based study. Ann Neurol. 1999;46:183-8.

7. Krex D, Klink B, Hartmann C, von DA, Pietsch T, Simon M, et al. Long-term survival with glioblastoma multiforme. Brain. 2007; 130:2596-606

8. Houillier C, Lejeune J, Ouaich-Amiel A, Laigle-Donadey F, Criniere E, Mokhtari K, et al. Prognostic impact of molecular markers in a series of 220 primary glioblastomas. Cancer. 2006;106:2218-23.

9. Shinojima N, Kochi M, Hamada J, Nakamura H, Yano S, Makino $\mathrm{K}$, et al. The influence of sex and the presence of giant cells on postoperative long-term survival in adult patients with supratentorial glioblastoma multiforme. J Neurosurg. 2004;101: 219-26.

10. Miller CR, Perry A. Glioblastoma. Arch Pathol Lab Med. 2007; 131:397-406.

11. Burton EC, Lamborn KR, Feuerstein BG, Prados M, Scott J, Forsyth $\mathrm{P}$, et al. Genetic aberrations defined by comparative genomic hybridization distinguish long-term from typical survivors of glioblastoma. Cancer Res. 2002;62:6205-10.

12. Burton EC, Lamborn KR, Forsyth P, Scott J, O'Campo J, UyeharaLock $\mathrm{J}$, et al. Aberrant $\mathrm{p} 53, \mathrm{mdm} 2$, and proliferation differ in glioblastomas from long-term compared with typical survivors. Clin Cancer Res. 2002;8:180-7.

13. Schmidt MC, Antweiler S, Urban N, Mueller W, Kuklik A, MeyerPuttlitz B, et al. Impact of genotype and morphology on the prognosis of glioblastoma. J Neuropathol Exp Neurol. 2002;61: 321-8.

14. Dehdashti AR, Sharma S, Laperriere N, Bernstein M. Coincidence vs cause: cure in three glioblastoma patients treated with brachytherapy. Can J Neurol Sci. 2007;34:339-42.

15. Bucy PC, Oberhill HR, Siqueira EB, Zimmerman HM, Jelsma RK. Cerebral glioblastomas can be cured! Neurosurgery. 1985;16: 714-7.

16. Burzynski SR, Lewy RI, Weaver R, Janicki T, Jurida G, Khan M, et al. Long-term survival and complete response of a patient with recurrent diffuse intrinsic brain stem glioblastoma multiforme. Integr Cancer Ther. 2004;3:257-61.

17. Cankovic M, Mikkelsen T, Rosenblum ML, Zarbo RJ. A simplified laboratory validated assay for MGMT promoter hypermethylation analysis of glioma specimens from formalin-fixed paraffin-embedded tissue. Lab Invest. 2007:87:392-7.

18. Ito M, Wakabayashi T, Natsume A, Hatano H, Fujii M, Yoshida J. Genetically heterogeneous glioblastoma recurring with disappearance of $1 \mathrm{p} / 19 \mathrm{q}$ losses: case report. Neurosurgery. 2007; 61:E168-9.

19. Kouwenhoven MC, Kros JM, French PJ, Biemond-ter Stege EM, Graveland WJ, Taphoorn MJ, et al. $1 \mathrm{p} / 19 \mathrm{q}$ loss within oligodendroglioma is predictive for response to first line temozolomide but not to salvage treatment. Eur J Cancer. 2006; 42:2499-503
20. Cairncross G, Berkey B, Shaw E, Jenkins R, Scheithauer B, Brachman D, et al. Phase III trial of chemotherapy plus radiotherapy compared with radiotherapy alone for pure and mixed anaplastic oligodendroglioma: Intergroup Radiation Therapy Oncology Group Trial 9402. J Clin Oncol. 2006;24: 2707-14.

21. Bauman GS, Ino Y, Ueki K, Zlatescu MC, Fisher BJ, Macdonald $\mathrm{DR}$, et al. Allelic loss of chromosome 1p and radiotherapy plus chemotherapy in patients with oligodendrogliomas. Int J Radiat Oncol Biol Phys. 2000;48:825-30.

22. Smith JS, Perry A, Borell TJ, Lee HK, O'Fallon J, Hosek SM, et al. Alterations of chromosome arms $1 p$ and $19 q$ as predictors of survival in oligodendrogliomas, astrocytomas, and mixed oligoastrocytomas. J Clin Oncol. 2000;18:636-45.

23. Brat DJ, Seiferheld WF, Perry A, Hammond EH, Murray KJ, Schulsinger AR, et al. Analysis of 1p, 19q, 9p, and 10q as prognostic markers for high-grade astrocytomas using fluorescence in situ hybridization on tissue microarrays from Radiation Therapy Oncology Group trials. Neuro Oncol. 2004;6: 96-103.

24. Nagasaka T, Gunji M, Hosokai N, Hayashi K, Ikeda H, Ito M, et al. FISH $1 \mathrm{p} / 19 \mathrm{q}$ deletion/imbalance for molecular subclassification of glioblastoma. Brain Tumor Pathol. 2007;24:1-5.

25. Boulay JL, Miserez AR, Zweifel C, Sivasankaran B, Kana V, Ghaffari A, et al. Loss of NOTCH2 positively predicts survival in subgroups of human glial brain tumors. PLoS ONE. 2007; 2:e576.

26. Nakagawa T, Ido K, Sakuma T, Takeuchi H, Sato K, Kubota T. Prognostic significance of the immunohistochemical expression of O-methylguanine-DNA methyltransferase, P-glycoprotein, and multidrug resistance protein-1 in glioblastomas. Neuropathology. 2008;29:379-88.

27. Mattos JP, Marenco HA, Campos JM, Faria AV, Queiroz LS, Borges $\mathrm{G}$, et al. Cerebellar glioblastoma multiforme in an adult. Arq Neuropsiquiatr. 2006;64:132-5.

28. Kopelson G. Cerebellar glioblastoma. Cancer. 1982;50:308-11.

29. Chamberlain MC, Silver P, Levin VA. Poorly differentiated gliomas of the cerebellum. A study of 18 patients. Cancer. 1990;65: $337-40$ 